\title{
Characteristic Features of Internal and External Faults for Use in Differential Protection
}

\author{
I.I. Litvinov \\ Faculty of Power Engineering, Power Stations Department \\ Novosibirsk State Technical University (NSTU) \\ Novosibirsk, Russia \\ litvinovii@mail.ru
}

\author{
A.A. Osintsev \\ Faculty of Power Engineering, Power Stations Department \\ Novosibirsk State Technical University (NSTU) \\ Novosibirsk, Russia \\ osintsev@list.ru
}

\author{
V.E. Glazirin \\ Faculty of Power Engineering, Power Stations Department \\ Novosibirsk State Technical University (NSTU) \\ Novosibirsk, Russia \\ glazirin.ve@power.nstu.ru
}

\begin{abstract}
The article aims to study the change of instantaneous values of differential current in power transformer protection circuits during magnetizing inrush when the unloaded transformer is connected to a power source and when a fault occurs in the protected area. Also, time behavior of the unbalance current in the generator, differential protection during remote faults is considered. Saturation of measuring current transformers during the fault transients leads to distortion of signals in their secondary windings, which can cause a long delay in the disconnection of the protected object or false operation of the protection if traditional protective algorithms are used. The peculiarities of the change in the instantaneous differential current should be considered when developing the protection algorithm because this allows recognizing faults before the first saturation of electromagnetic current transformers. For quick and correct recognition of internal faults of the power transformer, the authors proposed the control of the maximum value of the derivative of the differential current and the duration of its monotonous changing at the beginning of the transient process. As studies have shown, the parameters controlled can differ significantly under normal operation conditions and at the time when a fault occurs. The authors also showed that monitoring the time behavior of the unbalance current in the generator, differential protection during external remote faults is necessary to avoid false tripping. Mathematical simulation was used to study the magnetizing inrush, short-circuit faults, and transients in current transformer windings.
\end{abstract}

Keywords-transformer protection; generator protection; magnetizing inrush

\section{INTRODUCTION}

The use of digital technology in relay protection makes it possible to control work conditions of the protected equipment with greater precise, which should contribute to increasing the efficiency of the protection devices. The need for a fast and accurate determination of whether there is a fault within the protection zone or there is none is caused by an increase in the errors of the measuring current transformers (CT's) during fault transients, which can lead to both false tripping and intolerable delay in operation of the protective device. Therefore, when designing protective equipment, it is necessary to focus on the distinctive features of external and internal fault with which one can accurately judge about the presence of a fault within the protection zone even in the conditions of growth of the CT errors. This article deals with distinctive features of faults for use in the AC generator and differential protection of the power transformer.

Nowadays, many manufacturers of differential protection of the power transformer apply harmonic restrain to avoid tripping during magnetizing current inrush. This method makes protection sensitive to developing turn-to-turn faults in power transformers windings because pick-up current of differential relay can be reduced down to $0.3 I_{r}$ where $I_{r}$ is a power transformer rated current. Also, protective relaying with harmonic restrain properly refrains from tripping, CT should saturate during the transient process caused by an external fault $[1,2]$. However, if CTs with magnetic cores are used to supply this protective equipment, then intolerable delay in tripping or even failure to operate may happen because of CT's inaccuracies arising during fault transient. As it is shown in [3], if an internal fault leads to CT saturation (as a rule, if CT burden is correctly calculated and CT of a proper type is installed, then its saturation occurs only due to DC component of a transient fault current. Significant errors caused by the AC component of a fault steady-state current are to be avoided if a fault current is limited by power transformer reactance [4]), then the amplitude of higher harmonics raises so significantly that protective relay appears to be unable to distinguish between magnetizing current inrush and an internal fault until transient is over. This happens because the wave shape of a distorted fault current in CT secondary windings resembles one during the inrush. Based on foregoing, more effective algorithms of power transformer protection must be developed 
to eliminate intolerable delay in operation and provide reliable discrimination between an internal fault and magnetizing current inrush because use of measuring transformers without magnetic core is not always economically justified.

According to the fact that distortion of currents in differential circuits may cause confusion between faulty and normal state operation, it is desirable, wherever possible, to determine whether the protected object is faulty or not before the first saturation of CT's (i.e., when CT's work with appropriate accuracy). To achieve the objective, it is necessary to analyze the change in instantaneous values of differential currents and currents in CT's secondary windings to find distinctive features of faults that manifest before the first sign change of the derivative of the differential current. This is due to the fact that after this event the information about primary currents, generally speaking, is unreliable. As one can see from the example in Fig.1, the sign change of the derivative of the current in CT windings may indicate either its natural change (when magnetic core is not saturated and accuracy of the CT is sufficient, see curve 1), since fault current is a sine function with offset, or the point when distortion during the transient period starts (in other words, the moment of time when the CT saturates and its error increases, see curve 2).

As stated in [1, 2], an internal fault can be easily detected during the first half-cycle of a fault transient (i.e. before any of CTs experiences saturation). It is because for the first moments in time, after an internal fault occurs, one can observe a significant increase of instantaneous values of differential current and a relatively small increase of restraint current. Conversely, if one observes a relatively small increase of the differential current and a significant increase of the restrain current, then it is safe to say that an external fault occurred. Both of these rules are no longer observed if and when any of CTs are in the set saturates. Besides, the first rule cannot be applied directly for use in differential protection of the power transformer as magnetizing current inrush will cause relay protection to operate undesirably in accordance with this principle every time when a power transformer being protected is energized or its power supply is restored after clearing an external fault [5].

Consequently, it is necessary to find and study experimentally new and reliable distinctive features of faults and magnetizing current inrushes to quickly (precisely speaking, before the first sign change of the derivative of the differential current) recognize a fault if it occurs and selectively disconnect the protected object from the power grid.

Also, it should be noted that a fairly large number of scientific papers have been devoted to the analysis of the functioning of CTs during fault transients and in steady-state conditions. However, often fault transients were considered for the cases when a fault causes extremely large currents accompanied by a large DC component to flow through CT's primary windings. Such fault conditions lead to a deep saturation of the CTs cores and a significant distortion of the secondary currents [6]. Part of the technical solutions proposed in the mentioned papers, found application in present-day algorithms of digital differential protection.

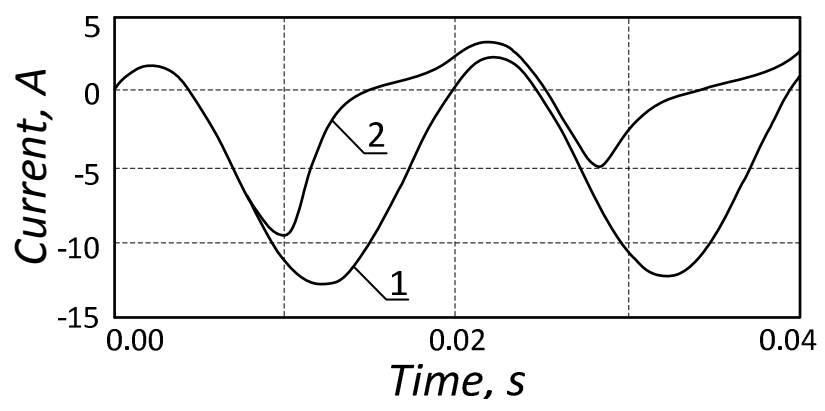

Fig. 1. Currents in CT windings under the conditions of saturation of the magnetic core. Curve 1 is the current in the primary CT winding (the primary current is referred to the secondary current). Curve 2 is the current in the secondary CT winging.

Behavior of differential protection during transients caused by remote external faults, accompanied by relatively small fault currents, has been studied to a much lesser extent. An example of a remote external fault is a short circuit fault on the power transmission line outgoing from a power station busbar. In this case, the generator current flowing to the fault location is commensurate with the value of the generator rated current, and the aperiodic time constant reaches $0.2-0.3 \mathrm{~s}$. If the fault occurred within the protection zone of time-delay protection, then the duration of such fault could reach $0.6 \mathrm{~s}$. The work features of the differential protection at the time when a remote fault occurs will be considered in the paper by the example of longitudinal differential protection of the AC generator.

\section{SPECIFICATION OF Distinctive FEATURES OF INTERNAL FAULTS, EXTERNAL FAULTS AND NORMAL OPERATION CONDITIONS}

\section{A. Power Transformer Protection}

To reliably recognize a fault at the very beginning of the transient process, the following features can be proposed. Cooperative use of the proposed methods together with conventional protective algorithms should improve the overall efficiency of power transformer differential protection since in all circumstances their use will not cause undesirable disconnection of the protected transformer from the grid during magnetizing current inrush and in the case of an external fault.

According to $[5,7,8]$, when a three-phase magnetizing current inrush takes place and large magnetizing currents flow through the primary windings of a delta-connected set of CTs (supposing we deal with a delta-wye power transformer and it is energized from the wye connected high voltage side), then the wave shape of the current in the relay's operating coil (i.e., differential current) is always identical to the offset and cut-off sine wave with width of the base up to 265 electric degrees. In general, a fault current is a complete sine wave biased along the axis of currents due to the presence of the DC component. For this reason, to detect internal faults, it is sufficient to control the duration of the increase (or decrease) of the differential current from the zero value to the instant of sign changing of its first derivative. If this duration exceeds the one 
maximum possible for differential current during magnetizing current inrush, which approximately, in accordance with [7], equals $7.36 \mathrm{~ms}$ (this corresponds to 132.5 electric degrees), it indicates an internal fault.

However, this characteristic feature may not appear in a fault differential current if CT's experience saturation during the first half-cycle since the beginning of a fault transient and so the sign change of the derivative of the controlled current occurs earlier than the indicated time. In addition, that prolonged monotonous growth of the module of the differential current is possible only if there is a significant DC component in a fault current (this feature can be demonstrated by the example given in Fig. 2, where the time of growth of the differential current in phase $\mathrm{A}$ is $8.2 \mathrm{~ms}$ ). If the $\mathrm{DC}$ component is absent, then the duration of monotonous growth of sine shaped current is less than $7.36 \mathrm{~ms}$. Therefore, this feature alone is not that effective, and it should be used in conjunction with the following one.

Firstly, let us determine ratio between peak values of a fault current and a magnetizing current during inrush. The maximum instantaneous value of a magnetizing current during inrush $i_{\text {inrush,max* }}$ (p.u.) can be obtained with use of the following formula $[5,7,9]$ :

$$
i_{\text {inrush }, \max *}=\frac{i_{\text {inrush }, \max }}{\sqrt{2} I_{r}}=\frac{U_{\max ^{*}}}{X_{T^{*}}}(1+A),
$$

where $i_{\text {inrush, max }}$ is the peak value of the magnetizing current during inrush (A); $I_{r}$ is the rated current of a given power transformer (A); $U_{\text {max* }}$ is the maximum value of the voltage of the power system to which the power transformer is connected (p.u.); $X_{T^{*}}$ is the equivalent inductive resistance of the power transformer being energized (p.u.); $A$ is the relative offset of the axis of sine wave of the flux linkage with respect to the inflection point of the saturation curve.

Equation (1) can be used to calculate peak values of inrush current for both single-phase and three-phase inrush provided that the parameter $X_{T^{*}}$ in the denominator of the formula (1) corresponds to the type of inrush (single-phase or three-phase) we want to consider.

In the case if a power transformer is energized from its high-voltage side, then the values of $X_{T^{*}}$ are always such [9] that under otherwise equal conditions, the value of $i_{\text {inrush,max* }}$ for single-phase and three-phase inrush is always less than the magnitude of the fault current caused by a short-circuit on the low-voltage side of a faulty transformer. This is due to the fact that demagnetization of the power transformer core is absent during the inrush, and therefore the inductive resistance limiting the peak inrush current is a sum of the leakage inductance measured at short-circuit in the secondary winding and the leakage inductance of the low-voltage winding measured at saturated core. For this reason, it can be expected that in the case of a fault the differential current rises at much faster rate than it does when magnetizing inrush occurs. It means there is a fundamental possibility to distinguish between magnetizing inrush and an internal fault by controlling the rate of change of the differential current, or, in other words, the absolute value of its derivative.
Obviously, to provide protection against interference and thus to avoid misoperation, it is necessary that the protective device utilizing the two proposed criteria does not operate unless instantaneous values of the differential current exceed a certain fixed value that determines the required noise immunity.

\section{B. Generator Protection}

Let us consider the behavior of differential protection installed on another equipment, namely, on a large-power AC generator with a split stator winding. Let us assume that CTs on the neutral side are installed into each of the parallel wires of all three phases (so there are $3 \mathrm{CT}$ s on the line-side and there are 6 CTs on the neutral-side). The CT's set in this case differs from the wye, so one will call such CT's set as double wye. A set of CT connected in the double wye operates in severer conditions compared to wye-connected CTs since the operating conditions of CTs connected in double-wye have a number of features.

First, a couple of so connected CTs installed in the same phase have common burden. To evaluate its influence, let us first consider an equation governing the processes in the secondary windings of wye-connected CTs:

$$
\frac{d \Psi_{Y j}}{d t}=L_{\sigma C T j} \frac{d i_{2 j}}{d t}+\left(R_{2 C T j}+R_{\text {load } j}\right) i_{2 j},
$$

where $\Psi_{Y}, i_{2}$ are magnetic linkage and the secondary current of a CT, respectively; $L_{\sigma C T}$ is the secondary winding leakage inductance of a CT; $R_{2 C T}, R_{\text {load }}$ are active resistance of the secondary winding of a CT and its load, respectively; $j$ indicates the number of the phase $(\mathrm{A}, \mathrm{B}$, or $\mathrm{C})$.

The equation governing the processes in the CT's connected in double wye is:

$$
\frac{d \Psi_{2 Y .1 j}}{d t}=L_{\sigma C T .1 j} \frac{d i_{2.1 j}}{d t}+\left(R_{2 C T .1 j}+R_{\text {loadj }}\right) i_{2.1 \mathrm{j}}+R_{\text {loadj }} \cdot i_{2.2 \mathrm{j}},
$$

where $i_{2.1 j}$ is the secondary current of the CT under consideration installed in the phase $j ; i_{2.2 j}$ is the secondary current of the parallel CT installed in the same phase.

When establishing equations (2) and (3), it was assumed that the CT's installed in the same phase have the same parameters, and the influence of other phases is absent, that is, the current in the neutral conductor is zero. It should be noted that such assumption can only be used for qualitative assessment of processes. One can clearly see from expression (3) that, in contrast with in wye-connected CTs, the total EMF put across the secondary winding of CTs connected in double wye is greater. This causes an increase in the flux linkage of so connected CTs compared to wye-connected CTs.

Secondly, the turn ratio of CTs connected in double wye and installed in the neutral-side of an $\mathrm{AC}$ generator is two times less than the turn ratio of CT's installed in linear-side of the generator. If one assumes that the cores of the CTs are made of the same material, that is, their magnetic characteristics are identical, then the induction in the magnetic cores of the CTs installed on the neutral-side is approximately 
twice as high as induction in CTs installed on the line-side. Hence, other things are equal in the presence of an DC component in the primary current. And if the same secondary currents are flowing in the CT's windings connected in wye and double wye, then the working induction in the magnetic cores of the latter CTs will reach the saturation faster, that is, the CTs connected in double wye will saturate earlier. That is why, the behavior of differential protection being supplied by that connected CTs is of particular interest.

\section{THEORETICAL STUdy OF THE PROPOSED FEATURES}

\section{A. Power Transformer protection}

Let us consider the nature of the change in the differential current during magnetizing current inrush and during an internal short-circuit fault through the example of a power transformer of type TDTs-125000/110. The nominal parameters of the given power transformer are as follows: rated voltage on the high-voltage side $U_{H V}=121 \mathrm{kV}$; rated current on the high-voltage side $I_{r}=600 \mathrm{~A}$; rated windings resistance $R_{T}=0.37 \mathrm{Ohms}$; short-circuit voltage $u_{s c}, \%=10.5 \%$ which corresponds to the reactance of $X_{T}=12.3 \mathrm{Ohms}$.

Assuming the power transformer is supplied from the high-voltage side, it is reasonable to consider only such internal faults that occur on the low-voltage size since the internal faults on high-voltage side can be easily detected by differential cutoff because in the latter case the fault currents are not limited by transformer impedance [1].

When simulating short-circuit currents, the DC component in one of the phases is taken as high as possible since only in this case a fast saturation of the CTs is possible. In accordance with [9], for all cases of magnetizing current inrushes, the parameter $A$ in equation (1) is taken to be the largest possible and equal to 0.39 . For the selected type of a power transformer the value of the equivalent reactance for single-phase inrush (the transformer is energized from the high-voltage side) $X_{T(1) \text { * }}$ is equal to 0.237 , the same parameter for three-phase inrush $X_{T(3) *}$ equals 0.35 , the value of $U_{\max }$ is assumed to be 1.0 .

Under these conditions, the maximum possible magnitude of the single-phase inrush $i_{\text {inrush, } \max (1)}$ equals (p.u.):

$$
i_{\text {inrush } \max (1)^{*}}=\frac{U_{\max ^{*}}}{X_{T(1)^{*}}}(1+A)=\frac{1 \cdot 1.39}{0.237}=5.87 .
$$

With the given value of $I_{r}=600 \mathrm{~A}$, one gets:

$$
i_{\text {inrush, } \max (1)}=i_{\text {inrush, } \max (1) *} I_{r} \sqrt{2} \approx 5,0 \mathrm{kA} .
$$

At the same time, the maximum possible magnitude of the three-phase inrush $i_{\text {inrush, } \max (3)}$ equals (p.u.):

$$
i_{\text {inrush } \max (3)^{*}}=\frac{U_{\max ^{*}}}{X_{T(3)^{*}}}(1+A)=\frac{1 \cdot 1.39}{0.35} \approx 4.0,
$$

hence,

$$
i_{\text {inrush } \max (3)^{*}}=i_{\text {inrush } \max (3)^{*}} I_{r} \sqrt{2} \approx 3.39 \mathrm{kA} .
$$

The effective value of the fault current in the case of threephase fault $I_{\text {faul }} *$ on the low-voltage side of the power transformer is (p.u.):

$$
I_{\text {fault }}=\frac{U_{\text {max* }}}{u_{s c, \%} / 100 \%}=\frac{1}{0,105}=9,52 .
$$

Consequently, the maximum instantaneous value of the fault current $i_{\text {fault }}$ equals (the fault current is referred to the high-voltage side of the power transformer):

$$
i_{\text {fault }}=I_{\text {fault }} I_{r} \sqrt{2}=9,52 \cdot 600 \sqrt{2}=8,08 \mathrm{kA} .
$$

The time constant of the aperiodic component of the fault current at the previously mentioned values of $R_{T}$ and $X_{T}$ is approximately $0.1 \mathrm{~s}$, and the corresponding value of the initial short-circuit current is $15.43 \mathrm{kA}$.

The following magnetizing inrushes are considered by means of simulation: 1) single-phase inrush with the peak value of $i_{\text {inrush, } \max (1)}$ in the first cycle; 2) type I and II three-phase inrushes [5] (with maximum periodic and aperiodic current in one of the three phases, respectively) with the peak value of $i_{\text {inrush, } \max (3)}$ in the first cycle. The mathematical model [10] was used to simulate magnetizing inrushes. When simulating a fault, initial short-circuit current was assumed to be equal to the one calculated above. Open circuit operation is taken as pre-fault conditions.

\section{B. Generator Protection}

Because of the fact that it is hard to predict time behavior of differential current and currents in secondary windings of CTs connected in double wye, the only way to study transients in primary and secondary circuits it is to carry out field tests or computer simulation. Investigation of remote short-circuits was carried out with use of MATLAB ${ }^{\circledR}$ software and the mathematical model of CTs connected in wye and double wye. The model of sets of CTs is based on equation (2) and (3) and principles of simulation of non-linear elements of a power grid [11]. The most important findings of multiple simulation results are to be presenter further.

\section{Simulation RESUltS}

\section{A. Power Transformer Protection}

The results of the simulation are shown in Figs. 2, 3, 4, 5 (for clarity, the figures show the differential current and its derivative only in one of three phases). It is shown that the maximum value of the rate of change of the differential current during magnetizing inrush does not exceed $1.3 \cdot 10^{6} \mathrm{~A} / \mathrm{s}$. At the same time, under the same conditions in the case of a fault the maximum value of the derivative is much higher and equals $4.3 \cdot 10^{6} \mathrm{~A} / \mathrm{s}$. Moreover, as early as fault transient starts, for the phase under consideration the value of the derivative equals $2.3 \cdot 10^{6} \mathrm{~A} / \mathrm{s}$ which already exceeds the maximum value of the derivative obtained for magnetizing inrush (for some phases in the case under consideration the maximum value of the derivative is reached at the very beginning of the fault transient). 
Thus, there is a fundamental possibility of detecting an internal fault at the very beginning of the fault transient, that is, before any of CTs experiences saturation (fast determination of a fault is facilitated by the fact that the derivative of the differential current reaches its peak value earlier than the differential signal itself). To do this, it is necessary to adjust pick-up of the protection so that is will not operate when derivative of the differential current at magnetizing inrush reaches its maximum value. Reproduction of magnetizing inrushes in different power transformers in order to determine the derivative of the differential current and select appropriate pick-up value can be carried out with the help of the mathematical model [10] and the guidelines for calculation of magnetizing current inrush [9].

Let us note that the proposed method is not so effective when a power transformer is energized from the low-voltage (medium-voltage) side. It is so since the amplitude value of the magnetizing current (as well as its derivative) during inrush in this case may turn out to be commensurable with the amplitude of a fault current, but this does not introduce significant complications since power transformers, as a rule, are switched on from the high-voltage side. At the same time, when the power transformer might be switched on from the low-voltage (medium-voltage) side, it seems advisable to suppress the action of the protection utilizing this feature. That is, protection should be allowed to operate only when differential current is former from secondary currents of CTs installed on the high-voltage side of a power transformer. Effective use of the proposed distinctive features can only be reached when false operation of the protection utilizing them is impossible. To provide selective work of protection in the process of external faults, when the transient unbalance current increases (the value of the unbalance current can be in the limit equal to the differential current at internal short-circuit [6], and therefore false tripping under these circumstances is possible), it is advisable to temporarily suppress the action of the protection until external fault is cleared. Exact registration of an external fault can be carried out according to the method described in $[1,2]$ when the external damage is fixed by the fact of a significant increase in the restraint current with a relatively small increase in the differential current in the first the moments of a fault transient.

The application of the proposed features allows the most severe faults in power transformers to be detected as quickly as possible. During off-peak conditions of the power system, the values of the derivative of the differential current during a fault can be commensurable with the derivative of the differential current during maximum magnetizing inrush, which reduces the effectiveness of the proposed method. However, a reduction in the fault currents reduces the probability of saturation of the CTs. Therefore, in this case a fault can be reliably determined by means of harmonic analysis. Thus, cooperative use of the conventional differential protection using harmonic restraint with the protection using proposed distinctive features of faults should be recommended.

\section{B. Generator Protection}

An example of the unbalance current of a remote fault is shown in Fig.6. This current is flowing through the AC generator circuits to the point of a fault. A characteristic feature of the unbalance current under consideration is its periodicity of the change which might cause false tripping of $\mathrm{AC}$ generator differential protection. By analyzing the oscillographs of secondary currents in CT's windings it is possible to detect a certain distinctive feature of the unbalance current which appears when a remote fault occurs. Namely, one can observe a continuous increase in the unbalance current (for 2 or more cycles) due to the saturation of a CT on one side of the $\mathrm{AC}$ generator and subsequent long attenuation due to the saturation of a CT of the same phase on the opposite side of the generator and/or due to the damping of the DC component of the primary fault current.

It is possible that after a gradual increase in the unbalance current and its subsequent smooth attenuation, this current increases again. Such an unbalance current behavior is typical only for large-power AC generators with a split stator winding in the case that CTs are installed in each neutral wire of a split phase separately. Let us describe the reasons for the mentioned behavior of the unbalance current. At the first moments, the growth of the unbalance current happens due to the saturation of the CT installed in one of the windings of the split stator phase. Then there is a subsequent decay of this signal due to the damping of the DC component of the primary current. Then the second increase in the unbalance current occurs due to the saturation of the second CT installed on the neutral side of the same phase. The final continuous attenuation of this signal is due to the saturation of the CT of the same phase, installed on the opposite side AC generator, and/or due to the damping of the DC component of the primary fault current. To detect this feature one has to control the differential current for a long period of time (for more than 1 cycle of a fault transient). In fact, in case of a nearby fault the described nature of the change in the unbalance current is similar, but in this case the interaction with other CTs in the CT's set significantly affects the distortion of the wave shape of the unbalance current. This leads to the fact that the indicated characteristic feature is not always observed under the conditions of a nearby fault. The most reasonable method of application of the distinctive feature found is to use it as an additional criterion for detecting external faults. For example, after detecting an external fault, some of the present-day protection devices temporarily suppress the operation of the protective algorithm for a certain time (for $100 \mathrm{~ms}$ or more) $[1,2]$ to avoid false tripping during external fault transient. However, as shows the example in Fig.6, the duration of an external fault may exceed the suppression time. Since the distortion of secondary currents is negligible, hence, its shape identical to a sine wave and its value is high enough to cause a false tripping. Consequently, the combination of these factors can result in false tripping after suppression time is expired. So, to avoid misoperation, it is necessary to apply the proposed feature, namely, if it is detected, it is necessary to reset the suppression time to the initial set value. 


\section{CONCLUSION}

Distinctive features of magnetizing inrush and internal faults for use in differential protection of the power transformer are proposed. The application of these features in differential protection of the power transformer makes it possible to improve the quality of the protection by reducing the time of recognition of a fault in a power transformer. It is advisable to use the proposed methods together with existing methods of power transformer protection. Also, a criterion was proposed to determine if a fault external to an $\mathrm{AC}$ generator is still present in the power grid. The criterion should be used to define the suppression time of generator differential protection to avoid false tripping during prolonged remote external fault.
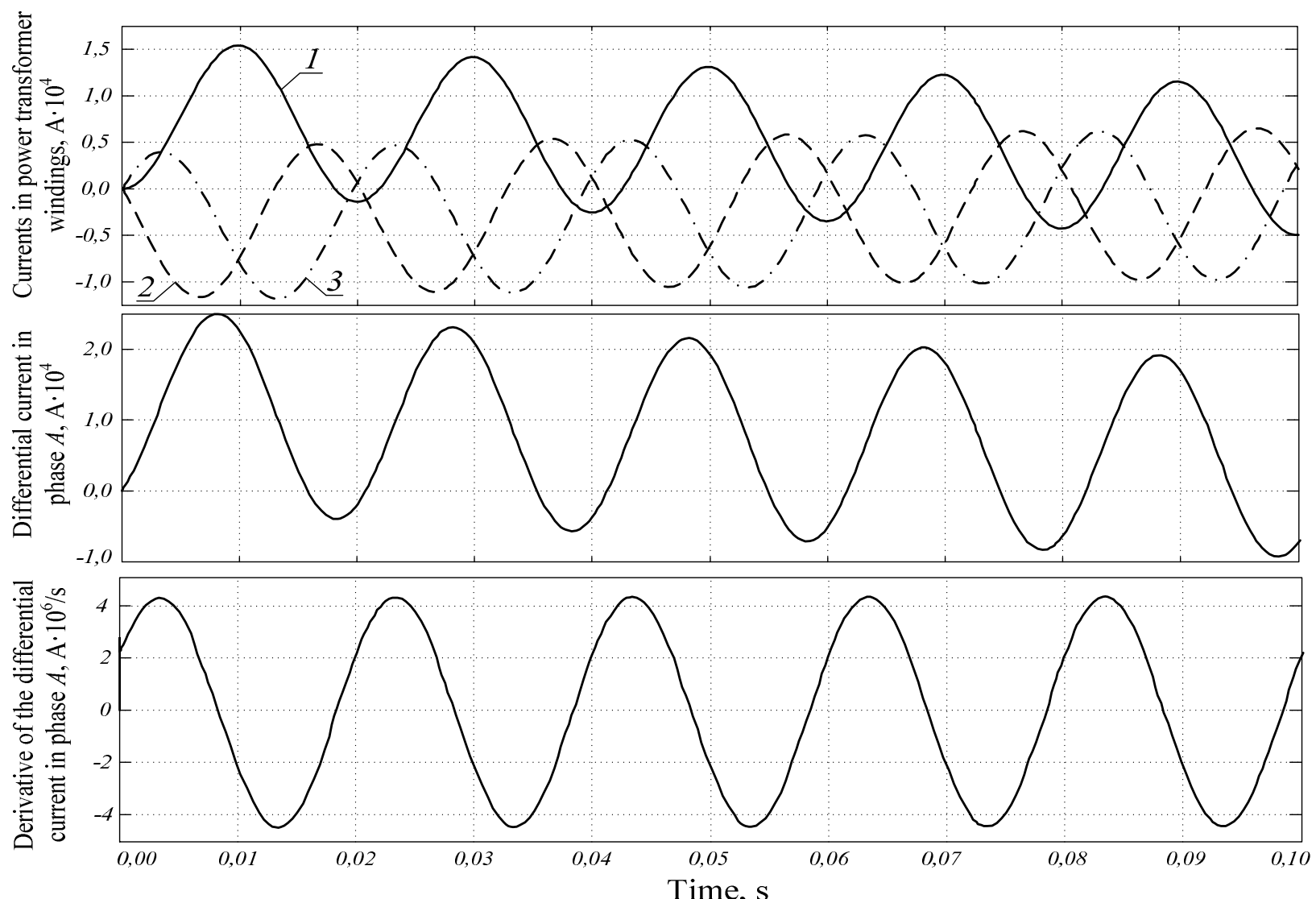

Fig. 2. Time behavior of fault currents in power transformer windings. Curves $1,2,3$ are fault currents in power transformer windings in phases A,B, and C, respectively. 


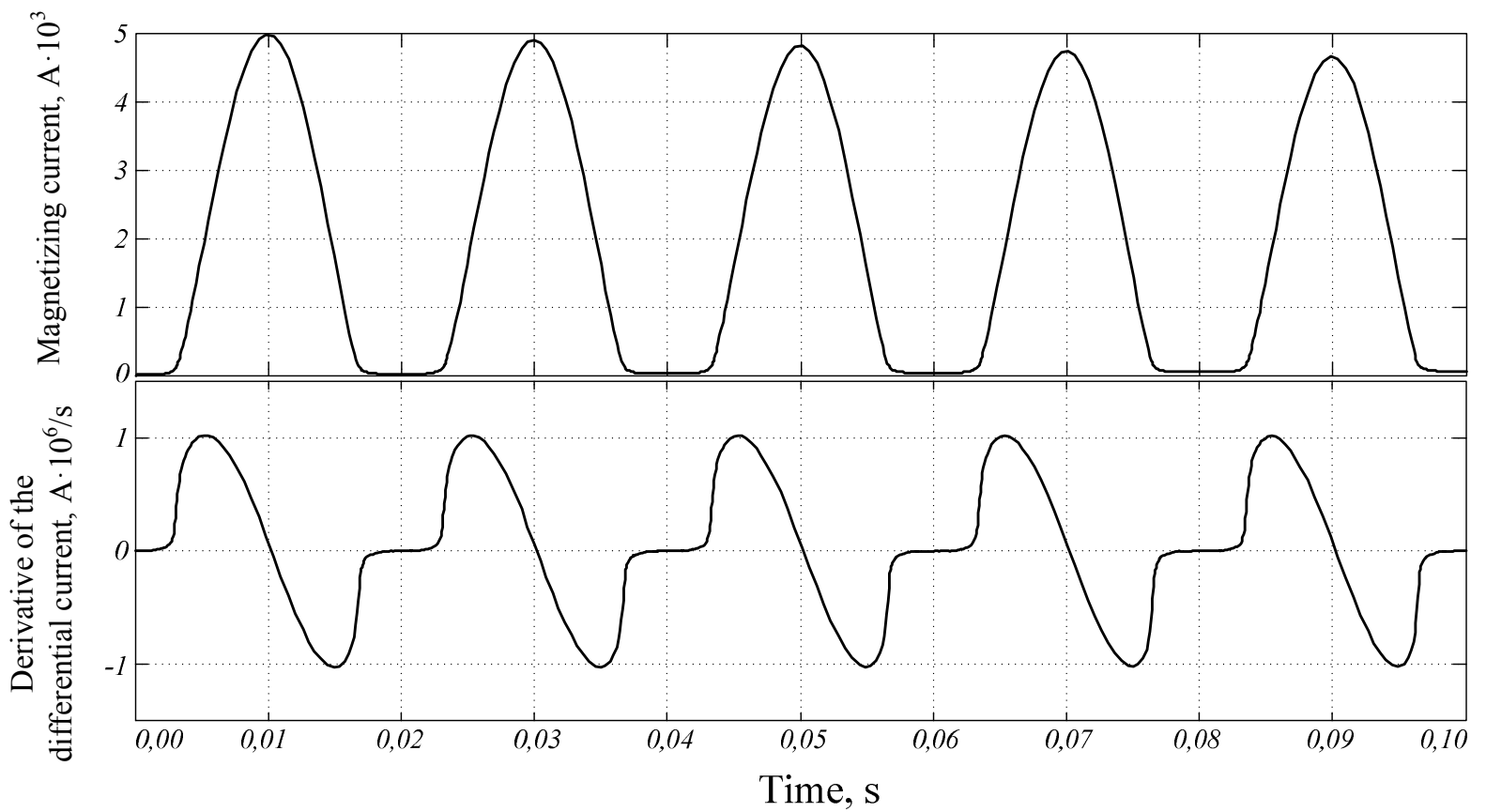

Fig. 3. Time behavior of magnetizing current of a power transformer during single-phase inrush

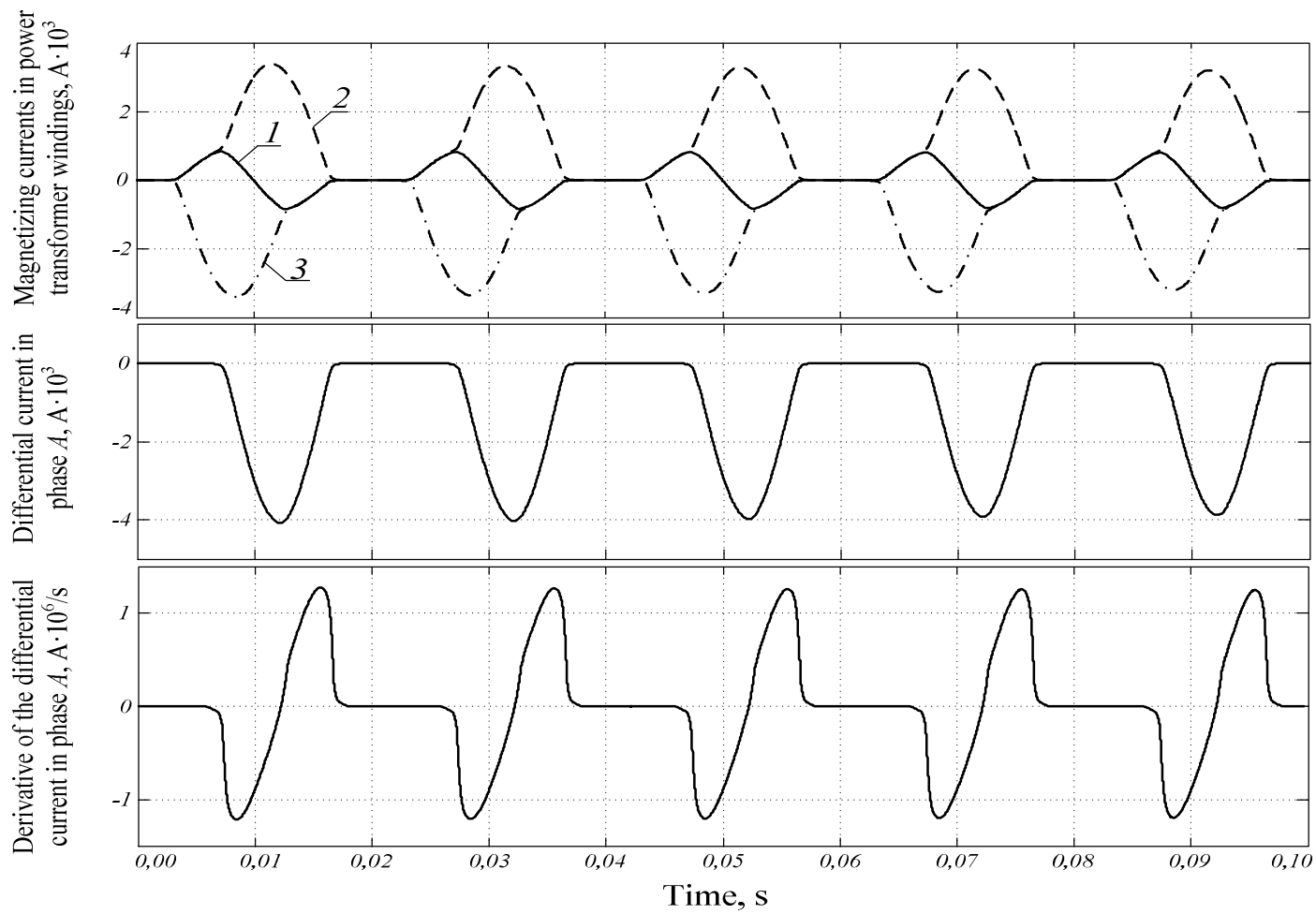

Fig. 4. Time behavior of magnetizing current of a power transformer during three-phase inrush of type I. Curves $1,2,3$ are power transformer magnetizing currents in phases $\mathrm{A}, \mathrm{B}$, and $\mathrm{C}$, respectively. 


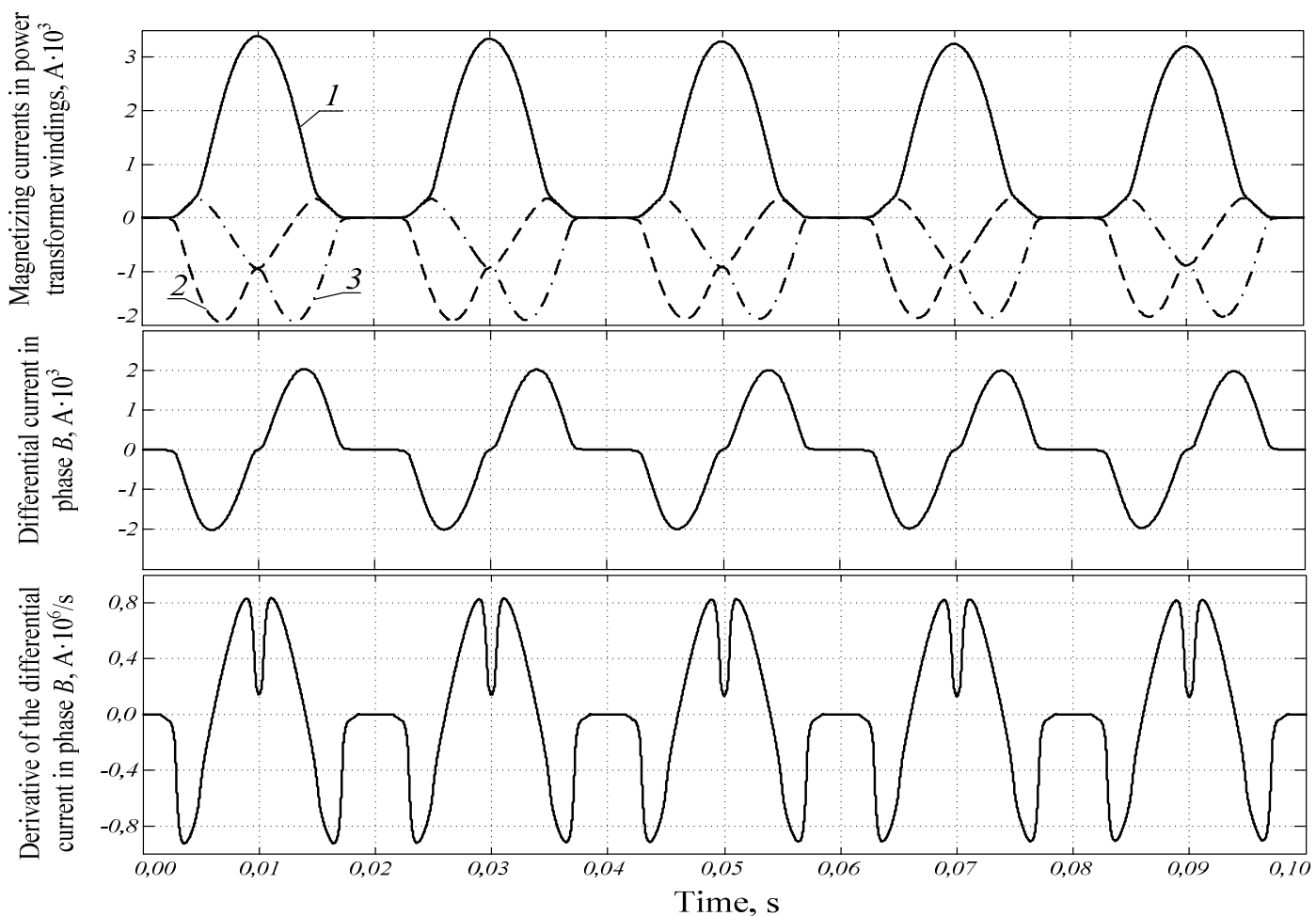

Fig. 5. Time behavior of magnetizing current of a power transformer during three-phase inrush of type I. Curves 1,2,3 are power transformer magnetizing currents in phases $\mathrm{A}, \mathrm{B}$, and $\mathrm{C}$, respectively.

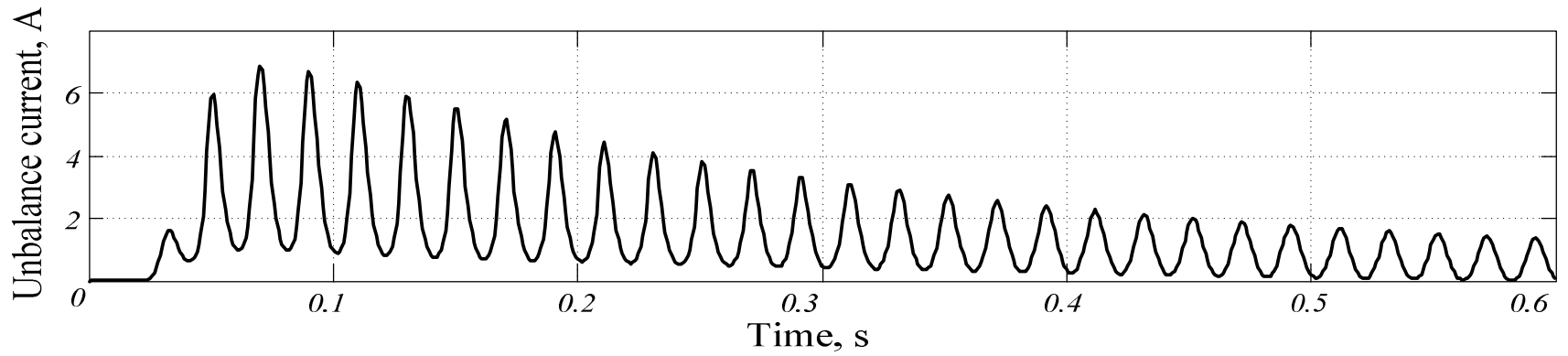

Fig. 6. An example of the curve of the unbalance current in differential protection of the AC generator during remote external three-phase fault

\section{References}

[1] G. Ziegler, Numerical Differential Protection: Principles and Applications, 1st ed. Wiley, 2005.

[2] E.M. Shneerson, Numerical Relay Protection, 2007.

[3] M.A. Kuparev, "Harmonic analysis of the currents in power transformer differential protection circuits in conditions of internal faults", ELEKTRO. Electrical engineering, electric power industry, electrotechnical industry, vol.3, pp. 15-18, 2007.

[4] E.P. Korolev, E.M. Liberson, Calculations of permissible loads in relay protection current circuits, 1980.

[5] A.S. Zasipkin, Differential protection of power transformers, 1989.

[6] L.V. Baginskiy, The main features of longitudinal differential protection of electrical equipment of power plants and substations, 2005.
[7] A.D. Drozdov, Electric circuits with ferromagnetic cores in relay protection, 1965.

[8] W.K. Sonnemann, C.L.Wagner, G.D. Rockefeller, "Magnetizing inrush phenomena in transformer banks", Power Apparatus and Systems, vol.38, pp. 884-892, October 1958.

[9] Guidelines for relay protection. Issue 12: Current zero sequence protection against earth faults in 110-500 kV lines. Calculations, 1980.

[10] I.I. Litvinov, V.E. Glazirin, "Development of a mathematical model of a power transformer when it is energized and determining the numerical parameters of the model", ELEKTRO. Electrical engineering, electric power industry, electrotechnical industry, vol.1, pp.18-24, 2017.

[11] V.E. Glazirin, G.E. Toropov, "Transient simulation in interconnected current transformers", Collection of scientific papers of NSTU, vol.3, pp.75-84, 2000. 